\title{
Classificação de risco em emergência - um desafio para as/os enfermeiras/os
}

Recebido em: 04/06/2010

Aceito em: 31/01/2011
Lícia Mara Brito Shiroma' Denise Elvira Pires de Pires ${ }^{2}$

Estudo descritivo e exploratório de abordagem qualitativa com o objetivo de conhecer a visão das/os enfermeiras/os acerca da implantação do Acolhimento com Avaliação e Classificação de Risco (AACR) em serviços de emergência. Os dados foram coletados através de entrevistas semiestruturadas com enfermeiras/os de um hospital universitário do sul do Brasil. Na voz das/os enfermeiras/os, AACR significa reorganização do atendimento e possibilidade de proporcionar melhor humanização, acesso e resposta satisfatória ao usuário em estado grave. Conclui que o AACR contribui para a agilidade e segurança no atendimento aos usuários e para diminuir a sobrecarga de trabalho da equipe.

Descritores: Humanização da Assistência, Sistemas de Saúde, Políticas Públicas, Acolhimento.

\section{Risk classification in emergency room - a challenge to nurses}

That is a descriptive and exploratory study of qualitative approach in order to know the nurses' point of view about the User Embracement with Evaluation and Risk Classification (AACR) in ER. Data were collected through semi-structured interviews with nurses of a university hospital in southern Brazil. In the voice of nurses, AACR means reorganization of care and to provide a better humanization, access and satisfactory response to the user in serious condition. It concludes that the AACR contributes to the agility and safety in patient care and decrease the workload of the team.

Descriptors: Humanization of Care, Health Systems, Public Policies, User Embracement.

\section{La clasificación del riesgo en el servicio de emergencia - un desafio para los enfermeros}

Se trata de estudio descriptivo y exploratorio con abordaje cualitativa con el objetivo de conocer la opinión de las enfermeras sobre la implantación de Acogimiento con la Evaluación y Classificación de Riesgos (AACR) en servicios de emergencia. Los datos fueron recolectados a través de entrevistas semi-estructuradas con enfermeras de un hospital universitario en el sur de Brasil. En la voz de las enfermeras, AACR significa la reorganización de la atención y la posibilidad de proporcionar mejor humanización, el acceso y la respuesta satisfactoria al usuario en estado grave. Se concluye que la AACR contribuye a la agilidad y la seguridad en el servicio al cliente y reducir la carga de trabajo del equipo.

Descriptores: Humanización de la Atención, Sistemas de Salud, Políticas Públicas, Acogimiento.

\section{INTRODUÇÃO}

A grande demanda de pacientes atendidos, a imprevisibilidade desses atendimentos, o espaço físico reduzido, a falta de priorização do atendimento, entre outros fatores, faz da emergência (EMG) um local com extrema dificuldade de organização do trabalho. Além disso, a busca indiscriminada pelos serviços de EMG mobiliza desnecessariamente recursos humanos e materiais especializados, reforça a desvalorização da atenção básica e soluciona, paliativamente, os problemas de saúde da população(1).

Dados estatísticos da instituição em estudo revelam que, nos últimosoitoanos, houvedecréscimononúmerodeatendimentos e acréscimo no número de pacientes internados na EMG, sendo constatado também maior número de internações de casos de maior gravidade. Nesse setor, são disponibilizados 13 leitos. No entanto, os registros de enfermagem acusam uma média de 25 a 30 usuários internados diariamente, permanecendo em cadeiras e macas nos corredores ${ }^{(2)}$.

Visando incorporar a estratégia de atendimento prevista no HumanizaSUS, o serviço de emergência passou por várias modificações, incluindo reforma da planta física da EMG, aquisição de equipamentos e mobiliários, sensibilização da equipe pelos consultores do Ministério da Saúde (MS) quanto ao Acolhimento com Avaliação e Classificação de Risco (AACR) e visita dos profissionais a instituições que já implantaram o AACR previsto na referida política.

O AACR é uma tecnologia utilizada pelo MS no sentido de reorientação da política assistencial nos serviços de emergência, articulando os valores de humanização e qualificação da assistência. A classificação de risco está fundamentada em conceitos internacionais estabelecidos pelo protocolo de Manchester, que prevê padrões para o atendimento nas emergências de modo a priorizar o atendimento de acordo coma

1 Enfermeira. Mestre em Enfermagem pelo PEN/UFSC. Chefe do Serviço de Emergência Adulto do Hospital Universitário da UFSC. Membro do Grupo de Estudos sobre Trabalho, Saúde, Cidadania e Enfermagem (Práxis). E-mail: Ibshiroma@yahoo.com.br.

2 Enfermeira. Doutora em Ciências Sociais/Unicamp. Professora do Departamento e Programa de Pós-Graduação em Enfermagem da Universidade Federal de Santa Catarina/ PEN/UFSC. Pesquisadora do CNPq. Presidente do Conselho Regional de Enfermagem-SC. E-mail: piresdp@yahoo.com. 
indicação clínica, com vista a superar os problemas encontrados no modelo tradicional de organização do atendimento por ordem de chegada. O protocolo de Manchester determina a classificação para prioridade de atendimento (de imediatotempo zero a não urgente - máximo de 240 minutos), sendo realizada pela equipe de enfermagem e especialmente pelas enfermeiras $^{(3)}$.

Desde 2007, foi implantado na EMG do hospital estudado o "acolhimento", realizado por acadêmicas de enfermagem. As mesmas conversam com os clientes que chegam, verificam sinais vitais e buscam ajuda da/o enfermeira/o de plantão quando detectam alterações que justifiquem a prioridade no atendimento.

No Brasil, a preocupação com a "humanização" surgiu vinculada à saúde da mulher e à humanização dos partos. Em 2000, o governo lançou o Programa Nacional de Humanização da Assistência Hospitalar (PNHAH), que passou a ser denominado Política Nacional de Humanização (PNH) em 2004, com o desafio de viabilizar os princípios do Sistema Único de Saúde (SUS) ${ }^{(4)}$.

Ressalta-se que, na Constituição Federal, aprovada em 1988, o capítulo dedicado à saúde retrata o resultado de todo o processo desenvolvido ao longo dos 20 anos, criando o SUS e determinando que "a saúde é direito de todos e dever do Estado", incluindo, portanto, os atendimentos de emergência.

Nesse sentido, um dos objetivos da PNH foi reestruturar os serviços de EMG, implantando o AACR, que associa o acolhimento com a classificação de risco, conforme o grau de sofrimento, o potencial de risco de vida ou agravos à saúde ${ }^{(4)}$.

Segundo o documento base da $\mathrm{PNH}$, acolhimento é definido como "uma postura ética, um modo de operar os processos de trabalho em saúde, de forma a atender todos que procuram os serviços de saúde, ouvindo seus pedidos e assumindo no serviço uma postura capaz de acolher, escutar e dar respostas mais adequadas aos usuários"(4).

A história da humanização do atendimento em saúde revela a existência de reflexões produzidas no âmbito da saúde, especialmente da enfermagem, desde o fim da década de $1950^{(5)}$.

Nesse contexto, a humanização é entendida como "valorização dos diferentes sujeitos implicados no processo de produção de saúde: usuários, trabalhadores e gestores. Os valores que norteiam essa política são a autonomia e o protagonismo dos sujeitos, a corresponsabilidade entre eles, o estabelecimento de vínculos solidários, a participação coletiva no processo de gestão e a indissociabilidade entre atenção e gestão"(4).

Os resultados esperados com a implantação do AACR nas emergências é a diminuição do risco de mortes evitáveis, extinção da triagem por funcionário não qualificado, priorização de acordo com critérios clínicos, obrigatoriedade de encaminhamento responsável do usuário a outro serviço quando houver necessidade, redução do tempo de espera, detecção de casos que se agravarão se o atendimento for postergado, melhoria da assistência nas emergências, redução da ansiedade e aumento da satisfação dos profissionais e usuários ${ }^{(6)}$.

Nesse contexto, a presente pesquisa teve por objetivo conhecer a visão das/os enfermeiras/os do serviço de EMG adulto de um hospital universitário acerca da implantação do AACR.

\section{METODOLOGIA}

Trata-se de uma pesquisa de campo descritiva e exploratória, com abordagem qualitativa, que utilizou entrevistas semiestruturadas para a identificação da percepção das/os enfermeiras/os acerca da proposta do MS de implantar o AACR. Os sujeitos do estudo foram oito enfermeiras/os, que correspondem a $89 \%$ do total desses profissionais lotados no referido serviço. Todas as/os enfermeiras/os foram convidadas/ os a participar da pesquisa e apenas uma enfermeira não foi incluída, pois se encontrava em licença médica. Os dados foram registrados utilizando gravador de voz. Todos assinaram o termo de consentimento livre e esclarecido. Para respeitar o anonimato, utilizou-se a abreviação "enf." seguida do número de ordem para especificação das falas.

O projeto foi aprovado pelo Comitê de Ética em Pesquisa com Seres Humanos da Universidade Federal de Santa Catarina, processo $n^{\circ}$ 084/08 FR 193325.

Para o tratamento dos dados, foi realizada a análise de conteúdo de Bardin, seguindo as três fases propostas pela autora. Préanálise, que consiste na fase de organização dos dados; exploração do material, que diz respeito a operações de codificação, classificação e categorização; e a terceira fase, de análise e interpretação referencial, na qual os dados "brutos" foram tratados de maneira a serem significativos e válidos ${ }^{(7)}$.

Processadas essas fases, foram compostas quatro categorias de análise: as concepções das/os enfermeiras/os do serviço de EMG acerca do AACR; expectativas de mudanças com a implantação do AACR; o protocolo de classificação de risco como instrumento de trabalho; acolhimento com acadêmicas de enfermagem.

\section{RESULTADOS E DISCUSSÃO}

\section{Contexto da pesquisa e perfil dos participantes}

A pesquisa foi desenvolvida no serviço de EMG adulto de um hospital geral, público, de ensino, em Santa Catarina. Esse serviço atende cerca de 300 a 350 pacientes por dia.

Analisando os dados pessoais dos entrevistados, nota-se que as/os enfermeiras/os da EMG possuem entre 25 e 39 anos, com predominância de mulheres $(85,72 \%)$. Trabalham em média há sete anos como enfermeiras/os e há menos de cinco 
anos na instituição e nesse serviço (71,43\%). Esse achado corresponde ao encontrado na literatura, que registra que a equipe dos serviços de EMG é formada por profissionais que recentemente entraram na instituição. Isso tem como vantagem um grupo com grande capacidade de trabalho, suportando bem a pressão e sobrecarga do serviço. Porém, a maior desvantagem é a falta de experiência ${ }^{(8)}$.

Aproximadamente a metade $(57,14 \%)$ das/os enfermeiras/os já trabalharam em outro serviço de EMG, demonstrando alguma experiência prática, porém nenhum possui especialização na área de urgência/emergência; 57,14\% participaram de algum curso preparatório e 42,86\% não possuem nenhuma formação específica na área.

\section{A concepção das/os enfermeiras/os do setor de EMG acerca de AACR}

Analisar as concepções das/os enfermeiras/os sobre AACR, conhecer suas expectativas e opiniões e ouvir as sugestões possibilitam saber onde se deve intervir e aprofundar as lutas da profissão pela qualificação da assistência de enfermagem, em especial no que diz respeito ao AACR, provendo subsídios para detectar os pontos que necessitam ser mais bem trabalhados com a equipe.

O AACR, no contexto da $\mathrm{PNH}$, para as/os enfermeiras/os daEMG, foi percebido como sendo um novo modelo de reorganização do serviço e atendimento humanizado prestado pela equipe, que consiste em atender o usuário que procura o serviço, avaliando suas queixas e necessidades e classificando-o para o atendimento de acordo com o grau de prioridade. Foi identificado como fundamental para melhorar a resolutividade e propiciar o fornecimento de respostas seguras aos usuários.

"Significa priorizar o atendimento de acordo com a gravidade com a qual a pessoa se apresenta na hora que vem procurar o atendimento de EMG. Isso seria a classificação de risco, e o acolhimento seria no sentido de direcionar essa pessoa para que ela já se sinta atendida desde sua chegada na porta da EMG. A classificação de risco, no meu ver, seria uma consequência desse acolhimento, que iria classificar de acordo com a gravidade, os sinais e sintomas que aquela pessoa está apresentando, para que ela possa ser atendida em tempo hábil para resolver o seu problema." (Enf. 7)

As/os enfermeiras/os consideram que existe relação entre acolhimento e classificação de risco, justificando que, durante a classificação de risco, você também está acolhendo o usuário, ouvindo suas queixas, dando respostas a seus questionamentos, orientando-o e educando-o.

Foi consenso entre as/os enfermeiras/os entrevistadas/os a implantação do AACR. Alguns aproveitaram o momento para colocar também suas frustrações em relação ao atendimento tradicional nas emergências.
"Sim, eu gostaria que fosse implantada. Uma vez eu lembro que uma paciente chegou e a acompanhante me falou: 'Ela está aqui desde as 10 horas da manhã, e ela estava com pressão alta desde as 9 horas.' Só que ela só conseguiu entrar para ser atendida às 16 horas e teve um AVC esperando." (Enf. 8)

$O$ que percebemos com a descrição é que o AACR pode significar não só a melhor ordenação do atendimento ao usuário, mas também evitar que problemas de saúde sejam agravados se o atendimento for protelado. Nesse sentido, corroboramos com o mencionado por autores que defendem a implantação do AACR. Segundo esses autores, o serviço de saúde assume sua função precípua de acolher e dar uma resposta positiva, capaz de resolver ou minimizar os problemas de saúde da população ${ }^{(1,9)}$.

\section{Expectativas de mudanças com a implantação do AACR}

Considerando a expectativa de implantação do AACR, perguntouse às/aos enfermeiras/os o que eles acham que mudaria para o usuário e para o serviço de EMG se fosse implantado o AACR. A percepção dos entrevistados quanto às mudanças para o usuário foi a possibilidade de reforçar as ações educativas no sentido de informação do que caracteriza o atendimento em emergência e a orientação para buscar a rede básica para atendimentos eletivos. Foi citado, também, que o AACR possibilita o atendimento ao usuário grave com mais agilidade e humanização, o que se constitui no foco central da assistência a ser prestada em serviços de EMG.

"Eu acho que o que mudaria é exatamente o foco de atendimento, pois o foco da EMG é atender situações emergenciais e quando está em risco a saúde do indivíduo. Quando tu consegues organizar esse serviço, tu acabas readquirindo a característica essencial do serviço. Acho que isso é uma característica realmente de reorganização, e daí, reorganizando o serviço, ele pode prestar assistência ao que ele se presta, que são situações emergenciais, situações que comprometem a vida do indivíduo." (Enf. 8)

As/os enfermeiras/os identificaram como mudanças positivas para o serviço de EMG agilidade no atendimento, diminuição da demanda e melhor organização do serviço. Quanto aos pontos negativos, nenhuma das/dos enfermeiras/os conseguiu identificar, talvez pela falta de vivência prática com o AACR.

A maioria (75\%) dos entrevistados considera que a/o profissional enfermeira/o é o mais habilitado para realizar a classificação de risco, justificando sua resposta baseada na associação de conhecimentos teórico-práticos, pelo maior manejo com sinais e sintomas, e não com diagnóstico em sua formação. Acrescentou, ainda, que a/o enfermeira/o está mais envolvida/o com políticas públicas e organização do ambiente de trabalho e mais preocupada/o com o acolhimento e a humanização do atendimento.

Em relação aos atributos da profissão de enfermagem, Pires refere que esse profissional domina um campo de 
conhecimentos que Ihe dá competência para cuidar do ser humano em todo o seu processo de viver, da concepção até a morte, em três dimensões: cuidar, educar e administrativo-

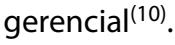

Duas/dois enfermeiras/os responderam que o profissional mais habilitado para realizar a classificação de risco seria o médico, baseando sua resposta no conhecimento científico que os últimos detêm e na responsabilidade legal da profissão médica.

\section{O protocolo de classificação de risco como instrumento de trabalho}

Os entrevistados avaliaram que o protocolo de classificação de risco sugerido pelo MS contempla a maior parte dos agravos de saúde atendidos na EMG, assim como auxilia na melhor avaliação e classificação do usuário. Afirmaram, ainda, que o mesmo dá embasamento, auxilia na padronização de condutas e tem respaldo legal. Mas deixaram claro que consideram a experiência em EMG o fator mais importante para realizar uma adequada classificação de risco.

\section{Acolhimento com acadêmicas/os de enfermagem}

As/os enfermeiras/os consideraram que o diálogo e a atenção que os acadêmicos dispensam aos usuários no "acolhimento" implantado no serviço estudado as/os tranquiliza, diminuindo consideravelmente os atritos e tumultos que aconteciam rotineiramente na recepção, assim como auxilia na organização do serviço. Esclarecem que, após sua implantação, a equipe ficou mais tranquila em saber que tem alguém da área de saúde observando os usuários que estão aguardando atendimento.

Essa concepção das/os enfermeiras/os encontra respaldo em Teixeira e colaboradores que entendem que o acolhimento pode significar não só a resolução dos problemas que o usuário apresenta, mas inclui uma escuta qualificada que valoriza as queixas e a identificação das necessidades ${ }^{(11)}$.

\section{CONSIDERAÇÕES FINAIS}

Destaca-se a contribuição que este estudo trouxe acerca do olhar das/os enfermeiras/os para a implantação do AACR em serviços de EMG. Os participantes do estudo percebem que a estratégia proposta pelo Ministério da Saúde contribui para melhorar a organização do serviço e a qualidade da assistência, ao mudar a lógica do atendimento de "ordem de chegada" para a de prioridade clínica, a qual propicia agilidade de acordo com as necessidades dos usuários, resultando em redução de riscos.

No entanto, a efetivação da mudança proposta pela política de humanização para a reorganização dos serviços de emergência não é algo tão fácil de ser conseguido. Muitas vezes, vai além dos limites e possibilidades da equipe, dependendo, também, de vontade política e das mudanças e condições estruturais dos serviços.

$\mathrm{O}$ estudo mostrou, ainda, que pequenas mudanças, como a implantação do "acolhimento" com acadêmicos de enfermagem, são importantes e que essas iniciativas, mesmo limitadas, contribuem para a melhoria da qualidade da assistência prestada.

O AACR representa hoje um desafio na consolidação de um modelo de atendimento de "porta aberta". Reconhecemse mudanças nas atitudes dos trabalhadores com maior preocupação com o atendimento humanizado e resposta às necessidades dos usuários. No entanto, pode-se afirmar que mobilizações isoladas, sem o apoio dos gestores, são insuficientes para maiores conquistas frente à demanda crescente, e cada vez mais complexa, por cuidados em saúde, principalmente do setor de EMG.

Ofertar uma assistência que proporcione atendimento com base nas melhores tecnologias disponíveis, com equipamentos e espaço físico adequados, conjugada ao reconhecimento das necessidades dos usuários e da equipe de saúde, continua sendo um grande desafio.

\section{Referências}

1. Cecilio LCO. Modelos tecno-assistenciais em saúde: da pirâmide ao círculo, uma possibilidade a ser explorada. Cad Saúde Pública. 1997; 13(3):469-78. 2. Boletim estatístico de atendimentos realizados na emergência adulto do hospital universitário Polydoro Ernani de São Thiago - Universidade Federal de Santa Catarina, 2010.

3. Mackway-Jones K, Marsden J, Windle J. Emergency triage: Manchester triage group. Massachussets: blackwell publishing, 2006.

4. Ministério da Saúde (BR). Cartilha da PNH. Brasilia: Ministério da Saúde, 2004. 5. Casate JC, Correa AK. Humanização do atendimento em saúde:

conhecimento veiculado na literatura brasileira de enfermagem.

Rev Latinoam Enferm, 2005; 13(1):105-11.

6. Ministério da Saúde (BR). Acolhimento com classificação de risco. Brasília:
Ministério da Saúde, 2007. Disponivel em:

http://portal.saude.gov.br/portal/saude/profissional/default.cfm

7. Bardin L. Análise de conteúdo. São Paulo: Martins Fontes, 2004.

8. Rodriguez JM. Emergências. Rio de Janeiro: MC Graw-hill, 2002.

9. Franco T B, Bueno WS, Merhy EE. O acolhimento e os processos de trabalho

em saúde: o caso de Betim-MG. Cad Saúde Pública. 1990; 15(2):121-31.

10. Pires DEP. A estrutura objetiva do trabalho em saúde. In: Leopardi MT et al.

Processo de trabalho em saúde: organização e subjetividade. Florianópolis, ed.

Papa-Livros, 1999.

11. Teixeira RA, Mishima SM, Pereira MJB. O trabalho da enfermagem

em atenção primária à saúde: assistência à saúde da família.

Rev Bras Enferm. 2000; 53(2):193-206. 